INPLASY

PROTOCOL

To cite: Morales et al. Physical exercise in natural environments and its influence on directed attention.

Education implication. A systematic review. Inplasy protocol 202130038. doi: 10.37766/inplasy2021.3.0038

Received: 13 March 2021

Published: 13 March 2021

Corresponding author:

Francisco Tomás González-

Fernández

francis.gonzalez.fernandez@gmail.com

Author Affiliation:

Pontificial University of Comillas.

CESAG. Mallorca.

Support: None declared.

Review Stage at time of this submission: Data extraction.

\section{Physical exercise in natural environments and its influence on directed attention. Education implication. A systematic review}

Morales, SB1; García-Taibo, O2; Baena-Extremera, $\mathrm{A}^{3}$; González-Fernández, FT4.

Review question / Objective: This systematic review aimed to: 1) Identify and summarize the studies that have examined the studies that had evaluated the performance of directed attention when physical exercise in natural environments was practiced.

Condition being studied: The effect of the physical exercise in natural environments on the directed attention in comparison with those performed in urban or built environments.

Information sources: Electronic databases (PubMed, Scopus, SPORTDiscus, and Web of Science) were searched for relevant publications prior to 20 February 2021.

INPLASY registration number: This protocol was registered with the International Platform of Registered Systematic Review and Meta-Analysis Protocols (INPLASY) on 13 March 2021 and was last updated on 13 March 2021 (registration number INPLASY202130038).

Conflicts of interest:

None declared.

\section{INTRODUCTION}

Review question / Objective: This systematic review aimed to: 1) Identify and summarize the studies that have examined the studies that had evaluated the performance of directed attention when physical exercise in natural environments was practiced.
Rationale: There is sufficient evidence to confirm that practice of physical exercise improves cognitive and emotional performance. Furthermore, the relationship of humans with nature seems to produce an improvement in different cognitive variables and specifically in directed attention. The mere presence of the human 
being in nature produces psychological, well-being and directed attention benefits. However, there is little research on the synergistic effect of physical exercise in natural environments, and how this affects directed attention, as a determining variable in academic performance.

Condition being studied: The effect of the physical exercise in natural environments on the directed attention in comparison with those performed in urban or built environments.

\section{METHODS}

Search strategy: Keywords and synonyms were entered in various combinations in the title, abstract or keywords: 1. Exercie Green 2. Physical exercise* natural environment 3. Directed attention 4.1 and 3 5. 2 and 3 6. Indoor or inside exercise 7. Exercise 8. 3 and 69.3 and 710.1 or 2 or 3 or 6 or 7.

Participant or population: All people from any age or sex, with normal vision, no partial/chronic injury or illness and no history of neuropsychological impairment.

Intervention: Exposure to nature was in outdoor environments containing natural elements or through virtual scenarios in nature.

Comparator: Similarity in training components in terms of volume, duration, intensity and mode. Physical exercise and directed attention test must be accurately determined.

Study designs to be included: We considered randomised crossover trials (RXT) or controlled/comparative trials (RCT), quasi-RXT and quasi-RCTS (acute and longitudinal trials were considered).

Eligibility criteria: Inclusion criteria: 1) All people from any age or sex, with normal vision, no partial/chronic injury or illness and no history of neuropsychological impairment. 2) RXT, RCT, quasy-RXT and quasy-RCTS. Exclusion criteria. 1) Other population with special conditions (e.g., injury, treatment, illness, diseases) 2) Other protocols.

Information sources: Electronic databases (PubMed, Scopus, SPORTDiscus, and Web of Science) were searched for relevant publications prior to 20 February 2021.

Main outcome(s): Any result comparing directed attention between environments.

Data management: A data extraction was prepared in Microsoft Excel sheet (Microsoft Corporation, Readmon, WA, USA) in accordance with the Cochrane Consumers and Communication Review Group's data extraction template. The Excel sheet was used to assess inclusion requirements and subsequently tested for all selected studies.

Quality assessment / Risk of bias analysis: A priori, the Strengthening the reporting of observational studies in epidemiology (STROBE) statement purposed by Von Elm et al. (2007) will be used, although depending on the characteristics of all included studies a more appropriate tool could be employed.

Strategy of data synthesis: Analysis and interpretation of results in this systematic review were only conducted in the case of at least study groups provided scientific evidence found in previous literature regarding the effect of Phisical Exercise or exercise in natural environment on Directed attention in the general population

Subgroup analysis: cognitive testing; phyisical and environmental testing.

Sensitivity analysis: To adjust for publication bias, a sensitivity analysis was conducted using the trim and fill method, with LO as the default estimator for the number of missing studies.

Language: English.

Country(ies) involved: Spain.

Other relevant information: There is sufficient evidence to confirm that practice 
of physical exercise improves cognitive and emotional performance. Furthermore, the relationship of humans with nature seems to produce an improvement in different cognitive variables and specifically in directed attention. The mere presence of the human being in nature produces psychological, well-being and directed attention benefits. However, there is little research on the synergistic effect of physical exercise in natural environments, and how this affects directed attention, as a determining variable in academic performance.

Keywords: directed attention; physical exercise; natural environment; exercise.

Contributions of each author:

Author 1 - Salvador Baena Morales - Lead the project. Performed the statistical analysis and report. Performed the search and methodological search and made the synthesis of results. Wrote and revised the manuscript.

Email: sbaena@um.es

Author 2 - Olalla García-Taibo - Performed the methodological search. Wrote and revised the manuscript.

Email: Otaibo@comillas.edu

Author 3 - Antonio Baena-Extremera Wrote and revised the manuscript.

Email: abaenaextrem@ugr.es

Author 4 - Francisco Tomás GonzálezFernández - Wrote and revised the manuscript.

Email: francis.gonzalez.fernandez@gmail.com 